\title{
Article \\ Transmitted Fetal Immune Response in Cases of SARS-CoV-2 Infections during Pregnancy
}

\author{
Ernesto González-Mesa ${ }^{1,2, *,+}+\mathbb{D}$, Eduardo García-Fuentes ${ }^{3,+}{ }^{\mathbb{D}}$, Rafael Carvia-Pontiasec ${ }^{4}$, \\ Ana I. Lavado-Fernández ${ }^{4}$, Celia Cuenca-Marín ${ }^{2}$, María Suárez-Arana ${ }^{2}$, Marta Blasco-Alonso ${ }^{2}$ (D), \\ Blanca Benítez-Lara ${ }^{1}{ }^{(D}$, Laura Mozas-Benítez ${ }^{1}$, Ana González-Cazorla ${ }^{1}$, Herink Egeberg-Neverdal ${ }^{1}$ \\ and Jesús S. Jiménez-López ${ }^{1,2} \mathbb{D}$
}

check for updates

Citation: González-Mesa, E.; García-Fuentes, E.; Carvia-Pontiasec, R.; Lavado-Fernández, A.I.; Cuenca-Marín, C.; Suárez-Arana, M.; Blasco-Alonso, M.; Benítez-Lara, B.; Mozas-Benítez, L.; González-Cazorla, A.; et al. Transmitted Fetal Immune Response in Cases of SARS-CoV-2 Infections during Pregnancy.

Diagnostics 2022, 12, 245. https:// doi.org/10.3390/

diagnostics12020245

Academic Editors: Paolo Ivo Cavoretto and Antonio Farina

Received: 2 January 2022

Accepted: 17 January 2022

Published: 19 January 2022

Publisher's Note: MDPI stays neutral with regard to jurisdictional claims in published maps and institutional affiliations.

Copyright: (C) 2022 by the authors Licensee MDPI, Basel, Switzerland. This article is an open access article distributed under the terms and conditions of the Creative Commons Attribution (CC BY) license (https:// creativecommons.org/licenses/by/ $4.0 /)$.
1 Biochemistry and Inmunology Department, Malaga Biomedical Research Institute-IBIMA, University of Málaga, Surgical Specialities, 29010 Málaga, Spain; blancabenl15@gmail.com (B.B.-L.); mozasbenitezlaura@gmail.com (L.M.-B.); anagonzalezcazorla2@gmail.com (A.G.-C.); Henrikegebergneverdal@gmail.com (H.E.-N.); jesuss.jimenez.sspa@juntadeandalucia.es (J.S.J.-L.)

2 Obstetrics and Gynecology Department, Málaga Regional Maternity Hospital (SSPA), 29010 Málaga, Spain; cecuman2@yahoo.es (C.C.-M.); dramariasuarez@gmail.com (M.S.-A.); martablascoalonso@gmail.com (M.B.-A.)

3 Digestive System Clinical Management Unit, Malaga Biomedical Research Institute-IBIMA, Virgen de la Victoria University Hospital, 29010 Málaga, Spain; edugf1@gmail.com

4 Provincial Unit of Pathological Anatomy of Malaga, Regional University Hospital of Malaga, SSPA, 29010 Málaga, Spain; resteban.carvia.sspa@juntadeandalucia.es (R.C.-P.); anabelap86@gmail.com (A.I.L.-F.)

* Correspondence: egonzalezmesa@uma.es

+ E.G.-M. and E.G.-F. contributed equally, and both should be considered as first authors.

Abstract: (1) Background: Little is known about the effects of SARS-CoV-2 on the placenta, and whether the maternal inflammatory response is transmitted vertically. This research aims to provide information about the effects of SARS-CoV-2 infection on maternal and fetal immunity. (2) Methods: We have studied placental changes and humoral and cellular immunity in maternal and umbilical cord blood (UCB) samples from a group of pregnant women delivering after the diagnosis of SARS-CoV-2 infection during pregnancy. IgG and IgM SARS-CoV-2 antibodies, Interleukin 1b (IL1b), Interleukin 6 (IL6), and gamma-Interferon (IFN- $\gamma$ ), have been studied in the UCB samples. Lymphocyte subsets were studied according to CD3, CD8, CD4, CD34, and invariant natural Killer T cells (iNKT) markers. We used in situ hybridization techniques for the detection of viral RNA in placentas. (3) Results: During the study period, 79 pregnant women and their corresponding newborns were recruited. The main gestational age at the time of delivery was 39.1 weeks (SD 1.3). We did not find traces of the SARS-CoV-2 virus RNA in any of the analyzed placental samples. Detectable concentrations of IgG anti-SARS-CoV-2 antibodies, IL1b, IL6, and IFN- $\gamma$, in UCB were found in all cases, but IgM antibodies anti-ARS-CoV-2 were systematically undetectable. We found significant correlations between fetal CD3+ mononuclear cells and UCB IgG concentrations. We also found significant correlations between $\mathrm{UCB}$ IgG concentrations and fetal CD3+/CD4+, as well as CD3+/CD8+ T cells subsets. We also discovered that fetal CD3+/CD8+ cell counts were significantly higher in those cases with placental infarctions. (4) Conclusion: we have not verified the placental transfer of SARS-CoV-2. However, we have discovered that a significant immune response is being transmitted to the fetus in cases of SARS-CoV-2 maternal infection.

Keywords: COVID-19 and pregnancy; SARS-CoV-2 infection; fetal lymphocyte subsets; fetal immune system

\section{Introduction}

Some previous studies revealed that pregnant women with different viral respiratory diseases were at high risk of developing obstetric complications and adverse perinatal 
outcomes related to changes in the immune response [1-3]. Most pregnant women who become infected by SARS-CoV-2 virus are generally asymptomatic or mildly symptomatic [4], but it is known that they are at increased risk of complications, severe morbidity, or mortality compared to the general population, as seen in other coronavirus infections [1,5-7], regardless of gestational age [8]. Pregnant women presenting with pneumonia or acute respiratory distress syndrome associated with COVID-19 have poorer obstetric and perinatal outcomes and a higher number of preterm cesarean sections [9]. Additionally, COVID-19 infection can cause adverse effects during pregnancy such as restricted fetal growth, preterm delivery, and perinatal mortality $[1,5-7,10]$. However, the exact mechanisms by which the infection produces these outcomes are not well known. While some studies assure that maternal hypoxemia caused by respiratory infection can be the cause of fetal hypoxia and the consequent complications of childbirth, others suggest that a direct effect of the virus on the placenta could be the cause of fetal morbidity and mortality [11-14]. COVID-19 and preeclampsia are strongly associated $[15,16]$, and both have been reported to have additive negative effects during the pregnancy [16]. Previous vascular conditions and COVID-19 inflammatory changes have been suggested as the main responsible factors $[15,16]$.

During pregnancy, many physiological changes occur, which have a significant impact on the immune, respiratory, cardiovascular, and blood clotting systems. The immune system adapts during pregnancy [17] to allow the growth of a semi-allogenic fetus, resulting in a probably altered immune response to infections. Pregnancy involves two immune challenges: establishing and maintaining tolerance to the fetus and, on the other hand, maintaining the ability to protect against microorganisms. In the first trimester, a proinflammatory state dictates necessary changes for the implantation of the zygote and development of the placenta; in the second trimester, it changes to an anti-inflammatory state to allow the fetus to grow; and in the third trimester, a pro-inflammatory state is reached again to prepare for childbirth $[18,19]$.

ACE-2 receptors are important in a SARS-CoV-2 virus tissue invasion, and these receptors have been described in placental tissue from all trimesters [20,21]. However, little is known of the effects of SARS-CoV-2 on the placenta [11-14]. Moreover, the possible transplacental transfer of the virus has not been fully clarified [22,23], although vertical transmission has been reported in some cases of COVID-19, complicating gestation [20,24,25]. Additionally, it is not clear whether the maternal inflammatory response to infection can be transmitted to the fetus through the placental pathway, inducing changes in the fetal immune system [26].

This research report provides information about the effects of SARS-CoV-2 infection on maternal and fetal immunity and about the correlations between them and placental changes. The objective of this work was to study if the effects of maternal inflammatory signals could be recognized in fetal immunity system.

\section{Materials and Methods}

This research was an observational and prospective study, carried out on pregnant women who were suffering at the moment of delivery or who had suffered from SARSCoV-2 infection throughout their pregnancy. The target population included any laboring woman with a positive SARS-CoV-2 PCR test either at hospital admission or at any time during the pregnancy. The study was performed in the Regional University Hospital of Málaga, within the Andalusian public health system (SSPA). Recruitment was carried out through consecutive sampling during the six months in which the study was active (from November 2020 to May 2021). The only exclusion criterion was the inability to give informed consent in the absence of a legal representative. All women invited to participate gave their consent.

\subsection{Ethical Aspects}

This study was carried out with respect for the fundamental rights and ethical postulates that affect biomedical research on human beings, following the international recom- 
mendations contained in the Declaration of Helsinki and its subsequent revisions. Likewise, the national recommendations have been followed in accordance with the national Biomedical Research Law 14/2007. The local ethical committee of Málaga authorized the research.

\subsection{Procedures}

Before the beginning of the study, sample collection kits were prepared, in which 3 tubes with EDTA were included for the cord blood samples, as well as a reservoir for the placentas with their corresponding anonymized identification by means of a code that allowed maintaining confidentiality. The samples were collected by midwives or obstetricians who attended the delivery. All the information related to the samples, as well as all the personal and clinical data associated with the samples, were considered confidential and were treated in accordance with the provisions of the General Data Protection Spanish regulations, Organic Law 3/2018 of 5 December regarding the protection of personal data and guarantee of digital rights, and Law 14/2007 of July 3 on biomedical research.

During the study period, the clinical protocols changed according to the regulations of the health authorities, although the PCR test in the nasopharyngeal exudate for the detection of the virus was systematically performed on all pregnant women who were admitted to hospital. Likewise, the clinical history recorded the possible incidents that occurred during pregnancy, including the positivity of the diagnostic tests for SARS-CoV-2 infection and the different clinical forms of presentation of COVID-19.

Once the participants' informed consent was obtained, at the time of delivery, the blood samples were collected and kept in a refrigerator at 15 degrees Celsius until the Biobank staff collected and processed them for their conservation at minus $80^{\circ} \mathrm{C}$. The samples were managed by the Biobank Provincial Málaga-IBIMA-SSPA, following standardized work protocols. The Malaga-IBIMA-SSPA Provincial Biobank is part of the Biobank of the Andalusian Public Health System (BSSPA) and the National Biobank Platform (exp. PT17/0015/0041). Blood samples were collected from the mother at the time of labor and from the umbilical cord immediately after birth. Placentas were stored at minus $30{ }^{\circ} \mathrm{C}$ until they were examined.

\subsection{Analysis}

The humoral immunity mediators IgG and IgM anti-SARS-CoV-2 antibodies, IL1b, IL6, and IFN- $\gamma$, were tested using commercial ELISA assays [27]. They are high sensibility and sensitivity tests, that have been tested for cross reactivity. In Table 1, the main characteristics of these tests are shown.

Table 1. Main properties of the tests.

\begin{tabular}{|c|c|c|c|c|}
\hline & $\begin{array}{l}\text { Minimum } \\
\text { Detectable } \\
\text { Dose }\end{array}$ & $\begin{array}{l}\text { Intra-Assay } \\
\text { Variation } \\
\text { Coefficient }\end{array}$ & $\begin{array}{l}\text { Inter-Assay } \\
\text { Variation } \\
\text { Coefficient }\end{array}$ & $\begin{array}{l}\text { Tested Absence of } \\
\text { Cross-Reactivity }\end{array}$ \\
\hline $\begin{array}{l}\text { RayBio }^{\circledR}{ }^{H} \text { Human IL-1 beta ELISA Kit }^{\circledR} \\
\quad\left(\text { RayBio }^{\circledR} \text { ref. ELH-IL1b) }\right.\end{array}$ & $0.3 \mathrm{pg} / \mathrm{mL}$ & $<10 \%$ & $<12 \%$ & $\begin{array}{l}\text { IL-1 } \alpha . \text { IL-1 } \beta . \text { IL-2. IL-4. } \\
\text { IL- } 8 . \text { IL-10. IL-12. IL-13. } \\
\text { IFN } \gamma \text { and TNF } \alpha\end{array}$ \\
\hline $\begin{array}{c}\text { Human IL-6 High } \\
\text { Sensitivity ELISA Kit (Diaclone. ref. } \\
\text { 950.035.096) }\end{array}$ & $0.81 \mathrm{pg} / \mathrm{mL}$ & $4.4 \%$ & $9.1 \%$ & $\begin{array}{l}\text { IL-1 } \alpha, \text { IL- } 1 \beta, \text { IL-2, IL-4, } \\
\text { IL-8, IL-10, IL-12, IL-12, } \\
\text { IFN } \gamma \text { y TNF } \alpha\end{array}$ \\
\hline $\begin{array}{l}\text { Human IFN } \gamma \text { High Sensitivity ELISA Kit } \\
\text { (Diaclone. ref. 850.900.096) }\end{array}$ & $0.69 \mathrm{pg} / \mathrm{ml}$ & $3.9 \%$ & $8.6 \%$ & $\begin{array}{l}\text { IL-1 } \alpha . \text { IL-2. IL-8. IL-12p40. } \\
\text { TNF } \alpha . \text { CD95/Fas. TRAIL. } \\
\text { ICAM-1. gp130 and } \\
\text { GM-CSF }\end{array}$ \\
\hline $\begin{array}{l}\text { Human SARS-CoV-2 Spike Protein S1 IgM } \\
\text { (MyBioSource. ref. MBS2614311) }\end{array}$ & $1.2 \mathrm{U} / \mathrm{mL}$ & $8 \%$ & $12 \%$ & IgM analogues \\
\hline $\begin{array}{c}\text { COVID-19 (SARS-CoV-2) quantitative IgG } \\
\text { ELISA (Demeditec. ref. } \\
\text { DECOV1901Q) }\end{array}$ & $3.6 \mathrm{AU} / \mathrm{mL}$ & $5.3 \%$ & $9.9 \%$ & IgG analogues \\
\hline
\end{tabular}


The placental tissue samples were processed for histological study. Finally, to detect viral RNA in placental tissue, we used in situ hybridization analysis. Commercial BOND RNAscope ${ }^{\circledR}$ Brown Detection [28] allows the visualization of target RNA molecules through chromogenic conversion.

\section{Results}

During the study period, 79 pregnant women and their corresponding newborns were recruited. All cases were simple pregnancies. The mean age of the participants was 31.9 years (SD 6.3). None of the participants had severe pneumonia that required admission to the ICU, and most of them (72.1\%) suffered from infection during the third trimester (mean gestational age at the time of infection 31.2 weeks (SD 8.3). The mean gestational age at the time of delivery was 39.1 weeks (SD 1.3). The prematurity rate in the participants was 5.1\%, and these cases consisted of four deliveries at week 36 . In most cases $(64.6 \%)$, the delivery started spontaneously, but the induction of labor was necessary in $26.6 \%$ of the cases. One of the cases was an elective caesarean section.

The reasons for induction were: 11 cases of premature rupture of the membranes $(13.9 \%), 7$ cases of fetal distress (8.9\%), and 4 prolonged pregnancies (over week 41 ). Regarding the newborns, $46.9 \%$ were female. The mean weight at birth was $3246 \mathrm{~g}$ (SD 438), so that only two newborns (2.6\%) had a weight under $2500 \mathrm{~g}$, and four (5.1\%) had weights above $4000 \mathrm{~g}$. The Apgar test scores were higher than 7 at the first and fifth minute in all cases, except for one of the newborns, who did not present any complications and could be discharged. Only three newborns required admission to the neonatal intensive care unit: one due to neonatal sepsis in a case of spontaneous delivery at 36 weeks in which the mother had been infected by SARS-CoV-2 in the second trimester with a mild form of the disease; another due to respiratory distress syndrome in a case of spontaneous delivery at 36 weeks with a positive SARS-CoV-2 test at admission for delivery; and a full-term newborn with an atrial septal defect. All three cases could be discharged in a healthy condition. The rest of the newborns did not present any complications until hospital discharge.

The analytical values corresponding to the hematological study in maternal blood are shown in the following Table 2 and Figure 1.

Table 2. Maternal blood cell count.

\begin{tabular}{|c|c|c|c|c|c|c|c|c|c|c|}
\hline & & $\mathrm{Hb}$ & WBC & Platelets & Neutrophils & Lymphocytes & Monocytes & Eosinophils & Basophils & $\begin{array}{l}\text { Granulocytes } \\
\text { Immature (\%) }\end{array}$ \\
\hline \multicolumn{2}{|c|}{ Mean } & 11.60 & 10.67 & 231.08 & 8.06 & 1.83 & 0.67 & 0.06 & 0.03 & 0.79 \\
\hline \multicolumn{2}{|c|}{ Std. Deviation } & 1.250 & 3.44 & 67.96 & 3.28 & 0.57 & 0.24 & 0.08 & 0.01 & 0.83 \\
\hline \multicolumn{2}{|c|}{ Minimum } & 7.90 & 4.79 & 99.00 & 3.18 & 0.77 & 0.33 & 0.00 & 0.01 & 0.03 \\
\hline \multicolumn{2}{|c|}{ Maximum } & 14.00 & 20.66 & 408.00 & 18.77 & 3.79 & 1.71 & 0.50 & 0.09 & 6.00 \\
\hline \multirow{3}{*}{ Percentiles } & 25 & 10.87 & 8.08 & 182.00 & 5.68 & 1.43 & 0.50 & 0.02 & 0.02 & 0.4 \\
\hline & 50 & 11.60 & 9.79 & 234.00 & 7.06 & 1.84 & 0.62 & 0.03 & 0.03 & 0.60 \\
\hline & 75 & 12.42 & 12.54 & 268.75 & 9.76 & 2.19 & 0.76 & 0.08 & 0.04 & 0.95 \\
\hline
\end{tabular}




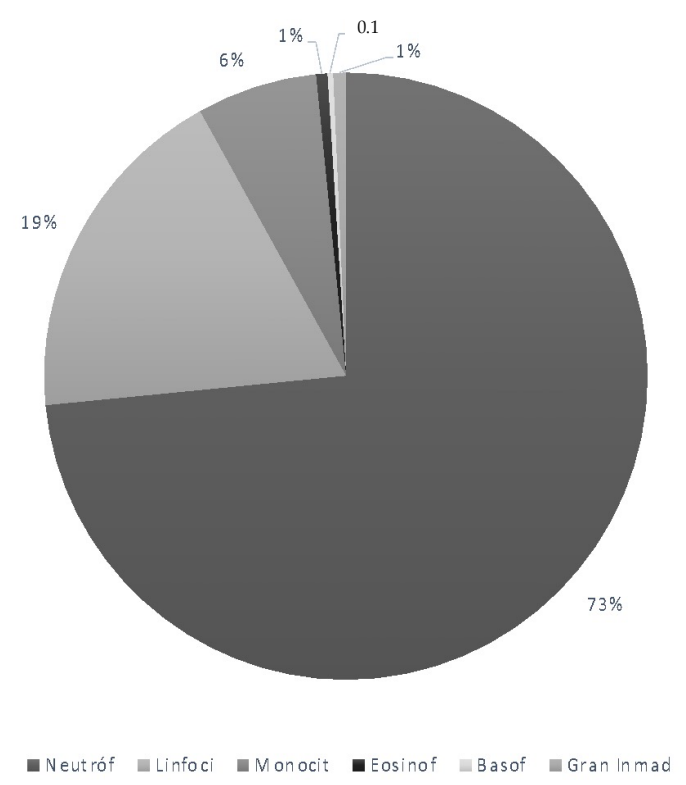

Figure 1. Maternal leucocyte subtypes.

The hematological values in umbilical cord blood are shown in Table 3 and Figure 2.

Table 3. Umbilical cord blood samples. Cell counts.

\begin{tabular}{|c|c|c|c|c|c|c|c|c|c|c|}
\hline & $\mathrm{Hb}$ & WBC & $\begin{array}{l}\text { Plate- } \\
\text { Lets }\end{array}$ & Neutrophils & Lymphocytes & Monocytes & Eosinophils & Basophils & $\begin{array}{c}\text { Granulocyte } \\
\text { Immature } \\
(\%)\end{array}$ & Erythroblasts \\
\hline Mean & 15.91 & 14.58 & 305.23 & 7.89 & 4.96 & 1.50 & 0.50 & 0.14 & 3.40 & 0.88 \\
\hline $\begin{array}{c}\text { Std. } \\
\text { Deviation }\end{array}$ & 1.67 & 4.04 & 70.55 & 3.06 & 2.46 & 0.71 & 0.38 & 0.20 & 2.44 & 1.45 \\
\hline Minimum & 12.80 & 8.20 & 34.00 & 2.56 & 2.78 & 0.69 & 0.00 & 0.01 & 0.08 & 0.00 \\
\hline Maximum & 22.80 & 25.55 & 470.00 & 18.00 & 20.30 & 5.30 & 2.50 & 1.50 & 9.60 & 9.10 \\
\hline $\begin{array}{c}\text { 25th } \\
\text { Percentile }\end{array}$ & 14.77 & 11.38 & 269.00 & 5.83 & 3.80 & 1.05 & 0.28 & 0.06 & 1.10 & 0.20 \\
\hline $\begin{array}{c}\text { 50th } \\
\text { Percentile }\end{array}$ & 15.75 & 14.24 & 300.00 & 7.14 & 4.36 & 1.41 & 0.42 & 0.10 & 3.00 & 0.40 \\
\hline $\begin{array}{c}\text { 75th } \\
\text { Percentile }\end{array}$ & 16.80 & 18.11 & 347.00 & 8.94 & 5.69 & 1.75 & 0.61 & .014 & 5.00 & 0.90 \\
\hline
\end{tabular}

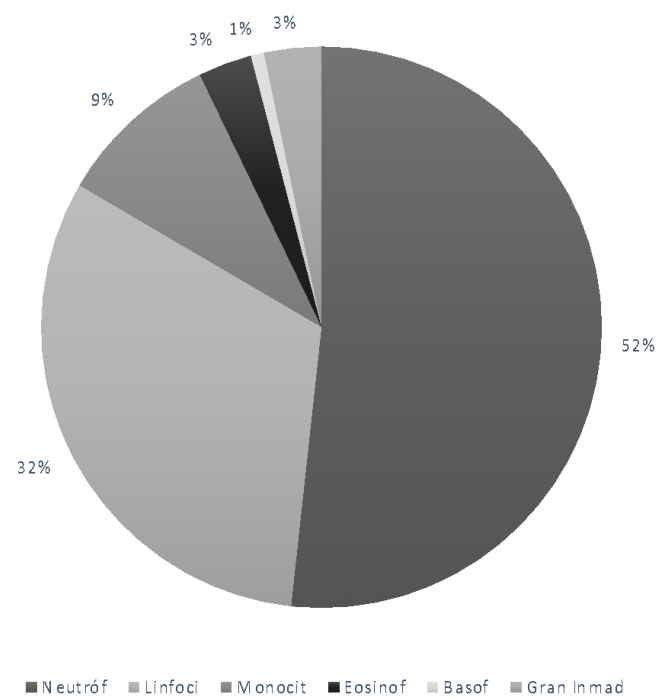

Figure 2. Umbilical cord blood samples. White blood cell subtypes. 
The mean values and corresponding standard deviations of IgG anti-SARS-CoV-2, IgM anti-SARS-CoV-2, IL6, IL1b, and IFN- $\gamma$ in umbilical cord blood are shown in the Table 4.

Table 4. Umbilical cord blood samples. Cytokines concentration.

\begin{tabular}{|c|c|c|c|c|c|c|}
\hline & & $\begin{array}{c}\text { Ab IgM } \\
(\mathrm{U} / \mathrm{mL})\end{array}$ & $\begin{array}{c}\text { Ab IgG } \\
(\mathrm{U} / \mathrm{mL})\end{array}$ & $\begin{array}{c}\text { IL-6 } \\
\text { (pg/mL) }\end{array}$ & $\begin{array}{c}\text { IL1B } \\
(\mathrm{pg} / \mathrm{mL})\end{array}$ & $\begin{array}{c}\text { IFN- } \gamma \\
(\mathrm{pg} / \mathrm{mL})\end{array}$ \\
\hline \multicolumn{2}{|c|}{ Mean } & 0.17 & 4227.91 & 11.53 & 0.58 & 7.02 \\
\hline \multicolumn{2}{|c|}{ Std. Deviation } & 0.36 & 5817.53 & 20.46 & 0.32 & 4.97 \\
\hline \multicolumn{2}{|c|}{ Minimum } & 0.00 & 108.2 & 2.1 & 0.3 & 0.7 \\
\hline \multicolumn{2}{|c|}{ Maximum } & 1.55 & 28348.6 & 109.1 & 1.9 & 18.8 \\
\hline \multirow{3}{*}{ Percentiles } & 25 & 0.00 & 246.81 & 3.18 & 0.43 & 1.56 \\
\hline & 50 & 0.00 & 1798.70 & 4.21 & 0.47 & 6.85 \\
\hline & 75 & 0.15 & 5871.07 & 7.29 & 0.55 & 10.64 \\
\hline
\end{tabular}

We observed statistically significant positive correlations between the time of evolution of the infection and the concentrations of IgG antibodies (Pearson's coefficient $\mathrm{r}=0.26$, $p<0.02$ ) and IFN- $\gamma$ (Pearson's coefficient $r=0.27, p<0.01$ ) in the UCB of the participants. Likewise, the concentrations of IL6 and IL1b were significantly correlated (Table 5).

Table 5. Umbilical cord blood samples. Correlations between cytokines concentrations. Pearson's coefficient. Pearson's significant correlation coefficients have been highlighted.

\begin{tabular}{cccccc}
\hline & $\begin{array}{c}\text { Time of } \\
\text { Evolution }\end{array}$ & $\begin{array}{c}\text { Ab IgG } \\
\text { (U/mL) }\end{array}$ & IL-6 (pg/mL) & $\begin{array}{c}\text { IL1B } \\
\text { (pg/mL) }\end{array}$ & $\begin{array}{c}\text { IFN- } \gamma \\
\text { (pg/mL) }\end{array}$ \\
\hline $\mathrm{Ab} \mathrm{IgG}(\mathrm{U} / \mathrm{mL})$ & $\mathbf{0 . 3 0 1 * *}$ & 1 & & & \\
$\mathrm{IL}-6(\mathrm{pg} / \mathrm{mL})$ & 0.053 & 0.002 & 1 & & \\
$\mathrm{IL} 1 \mathrm{~B}(\mathrm{pg} / \mathrm{mL})$ & -0.138 & 0.030 & $\mathbf{0 . 3 9 7 * *}$ & 1 & \\
$\mathrm{IFNg}(\mathrm{pg} / \mathrm{mL})$ & $\mathbf{0 . 2 8 0 *}$ & 0.064 & -0.013 & -0.083 & 1 \\
\hline ** & & & &
\end{tabular}

Table 6 shows the correlation of antibodies and elements of humoral immunity with the maternal blood cells. A significant correlation is observed between IL1b levels and the number of circulating eosinophils.

Table 6. Correlations between cytokines concentrations and maternal blood cells count. Pearson's significant correlation coefficients have been highlighted.

\begin{tabular}{|c|c|c|c|c|c|c|c|c|c|}
\hline & $\mathrm{Hb}$ & WBC & Platelets & Neutrophils & Lymphocytes & Monocytes & Eosinophils & Basophiles & $\begin{array}{c}\text { Granulocytes } \\
\text { Immature }\end{array}$ \\
\hline $\begin{array}{l}\mathrm{Ab} \text { IgG } \\
(\mathrm{U} / \mathrm{mL})\end{array}$ & -0.17 & 0.094 & 0.052 & 0.121 & -0.130 & 0.014 & -0.039 & -0.011 & -0.056 \\
\hline $\begin{array}{c}\text { IL-6 } \\
(\mathrm{pg} / \mathrm{mL})\end{array}$ & -0.08 & 0.037 & -0.084 & 0.055 & -0.090 & -0.001 & -0.037 & 0.109 & 0.071 \\
\hline $\begin{array}{c}\text { IL1B } \\
(\mathrm{pg} / \mathrm{mL})\end{array}$ & 0.00 & 0.017 & 0.051 & -0.002 & 0.095 & 0.027 & 0.336 * & 0.046 & -0.022 \\
\hline $\begin{array}{c}\text { IFN- } \gamma \\
(\mathrm{pg} / \mathrm{mL})\end{array}$ & 0.14 & 0.080 & 0.072 & 0.121 & -0.170 & -0.057 & -0.155 & 0.167 & 0.127 \\
\hline
\end{tabular}

* Significant $(p<0.05)$.

The correlations between the level of antibodies and other mediators of humoral immunity, and the counts of fetal blood cells are shown in Table 7.

We have observed that cases with very high IgG anti-SARS-CoV-2 concentrations (over the 75th percentile) were associated with higher fetal neutrophil counts and lower lymphocyte counts. Finally, in cases where the IFN- $\gamma$ concentrations were high (over the 75th percentile), the fetal neutrophil count was also higher. Table 8 shows the average values. 
Table 7. Correlations between cytokines concentrations and umbilical cord blood cells count. Pearson's coefficient. Pearson's significant correlation coefficients have been highlighted.

\begin{tabular}{|c|c|c|c|c|c|c|c|c|c|c|}
\hline & $\mathrm{Hb}$ & WBC & Platelets & Neutrophils & Lymphocytes & Monocytes & Eosinophils & Basophils & $\begin{array}{c}\text { Granulocytes } \\
\text { Immature } \\
(\%)\end{array}$ & Erythroblasts \\
\hline $\begin{array}{l}\mathrm{Ab} \text { IgG } \\
(\mathrm{U} / \mathrm{mL})\end{array}$ & 0.002 & -0.014 & 0.078 & 0.093 & 0.001 & 0.011 & -0.168 & 0.013 & 0.028 & 0.126 \\
\hline $\begin{array}{c}\text { IL-6 } \\
(\mathrm{pg} / \mathrm{mL})\end{array}$ & -0.111 & -0.085 & 0.155 & 0.167 & 0.006 & 0.094 & -0.103 & -0.027 & 0.146 & -0.111 \\
\hline $\begin{array}{c}\text { IL1B } \\
(\mathrm{pg} / \mathrm{mL})\end{array}$ & -0.005 & -0.058 & 0.009 & 0.002 & -0.181 & -0.125 & -0.144 & -0.205 & -0.066 & 0.111 \\
\hline $\begin{array}{c}\text { IFN- } \gamma \\
(\mathrm{pg} / \mathrm{mL})\end{array}$ & 0.111 & 0.020 & 0.261 * & 0.308 & -0.106 & 0.070 & 0.169 & -0.012 & $0.332^{\text {*^ }}$ & -0.029 \\
\hline
\end{tabular}

* Significant $(p<0.05)$.

Table 8. Main values in neutrophils and lymphocytes counts according to IgG concentration $(p<0.005)$.

\begin{tabular}{ccc}
\hline & Neutrophils & Lymphocytes \\
\hline IgG & & \\
$<75$ th Percentile & $7.88(3.1)$ & $4.96(2.6)$ \\
$>$ 75th Percentile & $7.94(2.93)$ & $4.95(1.6)$ \\
Interferon & & - \\
$<75$ th Percentile & $7.68(2.76)$ & - \\
$>75$ th Percentile & $8.82(4.18)$ & - \\
\hline
\end{tabular}

Regarding cellular immunity, Table 9 and Figure 3 show the proportion of cell subpopulations represented over the total mononuclear cells circulating in UCB. Table 9 shows the information on the subpopulations of T lymphocytes analyzed.

Table 9. Lymphocyte subset proportions.

\begin{tabular}{|c|c|c|c|c|c|c|}
\hline Subsets & Mean & Median & Std Dev & $p 25$ & $p 50$ & $p 75$ \\
\hline \% CD3+ & 42.52 & 40.15 & 12.948 & 31.55 & 40.15 & 53.675 \\
\hline$\%$ CD3+INKT+ & 1.695 & 1 & 1.7929 & 0.3 & 1 & 2.6 \\
\hline$\%$ CD3+INKT+CD34+ & 88.573 & 89.05 & 6.779 & 84.675 & 89.05 & 92.1 \\
\hline$\%$ CD3+CD4+ & 30.69 & 30.7 & 11.154 & 19.025 & 30.7 & 39.875 \\
\hline$\%$ CD3+CD4+CD34+ & 65.115 & 64.55 & 10.842 & 54.825 & 64.55 & 75.925 \\
\hline $\mathrm{CD} 3+\mathrm{CD} 8+$ & 11.708 & 11.1 & 4.497 & 8.225 & 11.1 & 14.375 \\
\hline$\%$ CD3+CD8+CD34+ & 35.132 & 32.25 & 14.446 & 23.8 & 32.25 & 45.7 \\
\hline Ratio CD4+/CD8+ & 2.874 & 2.67 & 1.198 & 2.199 & 2.674 & 3.555 \\
\hline Ratio CD4+CD34+/CD8+CD34+ & 2.089 & 2.006 & 0.667 & 1.59 & 2.00 & 2.44 \\
\hline
\end{tabular}

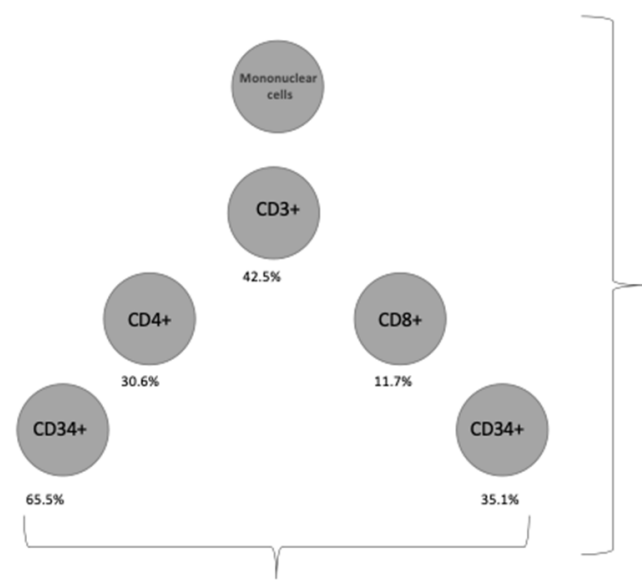

Figure 3. Cellular subsets. 
Table 10 shows the correlations between lymphocyte subpopulations in UCB and maternal hematological parameters. We have observed a negative and statistically significant correlation of the number of CD4+ T lymphocytes and the number of maternal neutrophils. Similarly, we have observed a negative correlation between the number of CD4+ and CD34+ $\mathrm{T}$ lymphocytes, and the number of circulating mature maternal granulocytes.

Table 10. Correlations between umbilical cord blood lymphocyte subsets and maternal blood cells count. Pearson's significant correlation coefficients have been highlighted.

\begin{tabular}{cccccccc}
\hline & CD3+ & $\begin{array}{c}\text { CD3+ } \\
\text { INKT+ }\end{array}$ & $\begin{array}{c}\text { CD3+INKT+ } \\
\text { CD34+ }\end{array}$ & $\begin{array}{c}\text { CD3+ } \\
\text { CD4+ }\end{array}$ & $\begin{array}{c}\text { CD3+CD4+ } \\
\text { CD34+ }\end{array}$ & $\begin{array}{c}\text { CD3+ } \\
\text { CD8+ }\end{array}$ & $\begin{array}{c}\text { CD3+CD8+ } \\
\text { CD34+ }\end{array}$ \\
\hline $\mathrm{Hb}$ & 0.140 & 0.044 & 0.014 & 0.165 & 0.065 & 0.012 & -0.021 \\
WBC & -0.310 & 0.120 & 0.069 & -0.426 & 0.036 & 0.132 & 0.080 \\
Platelets & -0.362 & 0.135 & -0.270 & -0.275 & -0.237 & -0.310 & 0.030 \\
Neutrophils & -0.329 & 0.094 & 0.118 & $-\mathbf{0 . 4 5 2 *}$ & 0.031 & 0.143 & 0.070 \\
Lymphocytes & -0.009 & 0.076 & -0.255 & 0.010 & -0.108 & -0.066 & -0.033 \\
Monocytes & -0.088 & 0.257 & 0.088 & -0.144 & 0.290 & 0.078 & 0.229 \\
Eosinophils & -0.034 & 0.089 & -0.138 & -0.070 & 0.184 & 0.075 & 0.237 \\
Basophils & -0.169 & 0.013 & -0.386 & -0.211 & -0.168 & 0.041 & -0.067 \\
Granulocytes & -0.189 & -0.253 & -0.385 & -0.176 & $-\mathbf{0 . 3 5 2 *}$ & -0.094 & -0.296 \\
\hline
\end{tabular}

* Significant $(p<0.05)$.

Table 11 shows the correlations between lymphocyte subpopulations in UCB and neonatal hematological parameters. We observed a negative and statistically significant correlation between the proportion of $\mathrm{T}$ lymphocytes and immature granulocytes. We also observed significant correlations between the proportion of iNKT+ cells and the number of neutrophils, lymphocytes, monocytes, eosinophils, and basophils circulating in UCB.

Table 11. Correlations between umbilical cord blood lymphocyte subsets and neonatal blood cells count. Pearson's significant correlation coefficients have been highlighted.

\begin{tabular}{cccccccc}
\hline & CD3+ & $\begin{array}{c}\text { CD3+ } \\
\text { INKT+ }\end{array}$ & $\begin{array}{c}\text { CD3+INKT+ } \\
\text { CD34+ }\end{array}$ & $\begin{array}{c}\text { CD3+ } \\
\text { CD4+ }\end{array}$ & $\begin{array}{c}\text { CD3+CD4+ } \\
\text { CD34+ }\end{array}$ & $\begin{array}{c}\text { CD3+ } \\
\text { CD8+ }\end{array}$ & $\begin{array}{c}\text { CD3+CD8+ } \\
\text { CD34+ }\end{array}$ \\
\hline Hb & 0.048 & -0.069 & 0.299 & 0.096 & 0.199 & -0.122 & 0.088 \\
WBC & -0.117 & 0.033 & -0.068 & -0.158 & -0.131 & 0.038 & -0.004 \\
Platelets & -0.268 & 0.335 & -0.101 & -0.199 & 0.126 & -0.281 & 0.107 \\
Neutrophils & -0.247 & $\mathbf{0 . 4 4 5 *}$ & -0.214 & -0.186 & 0.084 & -0.229 & 0.201 \\
Lymphocytes & -0.251 & $\mathbf{0 . 5 8 1} * *$ & -0.238 & -0.188 & 0.246 & -0.238 & 0.287 \\
Monocytes & -0.237 & $\mathbf{0 . 5 6 8} * *$ & -0.225 & -0.178 & 0.219 & -0.221 & 0.303 \\
Eosinophils & -0.108 & $\mathbf{0 . 4 3 7 ^ { * }}$ & -0.059 & -0.085 & 0.322 & -0.086 & 0.307 \\
Basophils & -0.289 & $\mathbf{0 . 6 0 7 *}$ & -0.266 & -0.238 & 0.150 & -0.211 & 0.311 \\
Granulocytes & -0.315 & 0.272 & 0.123 & -0.280 & 0.178 & -0.239 & 0.152 \\
Erythroblasts & $-\mathbf{0 . 3 8 1 *}$ & 0.210 & -0.025 & -0.301 & -0.171 & -0.338 & -0.039 \\
\hline
\end{tabular}

* Significant $(p<0.05) ;{ }^{* *}$ significant $(p<0.01)$.

UCB circulating IgG levels show significant correlation with the proportion of $\mathrm{T}$ lymphocytes in the mononuclear cell population and, specifically, with the CD4+ and CD8+ lymphocyte subpopulations. The CD8+/CD34+cell subpopulation and the iNKT subpopulation show significant correlation with IL1b concentrations (Table 12). 
Table 12. Correlations between umbilical cord blood lymphocyte subsets and umbilical cord blood cytokines concentrations. Pearson's significant correlation coefficients have been highlighted.

\begin{tabular}{cccccccc}
\hline & CD3+ & $\begin{array}{c}\text { CD3+ } \\
\text { INKT+ }\end{array}$ & $\begin{array}{c}\text { CD3+INKT+ } \\
\text { CD34+ }\end{array}$ & $\begin{array}{c}\text { CD3+ } \\
\text { CD4+ }\end{array}$ & $\begin{array}{c}\text { CD3+CD4+ } \\
\text { CD34+ }\end{array}$ & $\begin{array}{c}\text { CD3+ } \\
\text { CD8+ }\end{array}$ & $\begin{array}{c}\text { CD3+CD8+ } \\
\text { CD34+ }\end{array}$ \\
\hline $\begin{array}{c}\mathrm{Ab} \mathrm{IgG} \\
(\mathrm{U} / \mathrm{mL})\end{array}$ & $\mathbf{0 . 4 3 4}{ }^{* *}$ & 0.079 & 0.069 & $\mathbf{0 . 3 1 6 *}$ & 0.217 & $\mathbf{0 . 4 8 1} * *$ & 0.156 \\
$\mathrm{IL}-6(\mathrm{pg} / \mathrm{mL})$ & -0.047 & -0.062 & -0.028 & -0.074 & -0.128 & 0.047 & -0.139 \\
$\begin{array}{c}\mathrm{IL} 1 \mathrm{~B} \\
(\mathrm{pg} / \mathrm{mL})\end{array}$ & -0.264 & $\mathbf{0 . 4 6 0 * *}$ & -0.186 & -0.218 & 0.105 & -0.213 & $\mathbf{0 . 3 3 3} *$ \\
\hline
\end{tabular}

* Significant $(p<0.05) ;{ }^{* *}$ significant $(p<0.01)$.

We observed a negative correlation between fetal weight and the proportion of CD3+ lymphocytes $(\mathrm{r}=-0.33, p=0.05)$ and $\mathrm{CD} 4+$ lymphocytes $(\mathrm{r}=-0.33, p=0.037)$; however, we did not find any relationship between the different cell subpopulations and gestational age at the time of delivery, maternal age, type of delivery, form of delivery onset, or the trimester of infection.

We found a higher proportion of CD3+/CD8+/CD34+ lymphocytes in cases in which the placenta showed infarction areas (mean 32.8, SD 14.05, versus 44.27 SD 12.99, $p=0.044$ ). On the other hand, the observed proportion of CD3+/CD8+ lymphocytes was significantly higher in cases in which SARS-CoV-2 infection had evolved over two weeks, compared to cases in which the diagnosis was in the $24 \mathrm{~h}$ prior to delivery (mean $9.79 \mathrm{SD}=3.0$, compared to 13.73 . $\mathrm{SD}=4.9 ; \mathrm{F}=7.66 ; p=0.009)$.

\section{Placental Study}

We found no RNA of the virus in the placental tissue analyzed until this time, nor clear evidence of the transplacental passage of the virus. However, we observed the existence of placental changes that could be related to the effects of SARS-CoV-2 infection, especially of the ischemic type, pointing to the existence of vascular accidents in placental microcirculation.

\section{Discussion}

In this research report, we have studied placental changes and humoral and cellular immunity in maternal and umbilical cord blood (UCB) samples from a group of pregnant women delivering after a diagnosis of SARS-CoV-2 infection during pregnancy.

Regarding fetal transmission of SARS-CoV-2, our in situ hybridization (RNA scope) analysis failed in the detection of RNA traces. Moreover, newborns had negative PCR analyses in all cases. Although the existence of receptors in trophoblastic tissue has been described $[24,29,30]$, and it is known that trophoblastic cells can produce type II transmembrane serine-protease (the main mediator of the entry of the virus into cells), our results show that, at least in our samples, the placenta has not been a specific target organ for the virus.

It should be considered that the expression of ACE-II and type II transmembrane serine-protease receptors in third-trimester placentas might be less relevant [31], making vertical transmission pathway unimportant and reserved for cases in which some gestational conditions related to the renin-angiotensin axis could modify the expression of ACE-II receptors or facilitate other routes of access to the fetal compartment. Our sample of pregnant women in the third trimester, who, in general, had no special risk factors, demonstrated results that would support this hypothesis.

On the other hand, pathological analysis showed signs of placental vascular malperfusion (mainly placental infarctions and retroplacental hemorrhages) in $37.2 \%$ of cases, similar to those described in previous studies [32].

Some previous studies showed a lack of transplacental passage of pro-inflammatory cytokines [33,34], so the finding of detectable concentrations of IL- $1 \mathrm{~b}$, IL-6, and IFN- $\gamma$ are most likely related to the triggering of a certain fetal inflammatory response. In fact, IFN- $\gamma$ 
concentrations correlated with WBC count in the newborn, indicating the existence of neonatal immune reactivity. The placental transfer of immunoglobulin has been previously reported [34,35], especially IgG, and to a lesser extent, IgM [35]. In the sample that we have studied, we detected antibodies of the IgG type in the umbilical cord blood in increasing concentrations as the time of the evolution of the disease increased.

At the time of writing this research report, we studied lymphocyte subsets from 44 of the recruited cases. We observed that $42.5 \%$ of mononuclear cells express CD3, corresponding to T lymphocytes. The ratio of CD4/CD8 lymphocytes was 2.8 (SD 1.19). Several findings support our research hypothesis regarding the existence of a cellular immunity response transmitted to the fetus. Firstly, the observed relationship between the concentration of anti-SARS-CoV-2 IgG antibody and the cellular count of CD3+ lymphocytes points towards the existence of a fetal cellular response, with an increase in the production of T lymphocytes. Moreover, within CD3+ lymphocytes, an increase in CD4+ and CD8+ lymphocytes counts has been observed, with a predominance of CD8, as anti-SARS-CoV-2 IgG levels increase. These findings are in line with previous reports about the increased capacity of fetal T-cell subsets to cytokine stimulation [36]. Finally, pathological analysis of placentas showed that signs of poor perfusion (placental infarctions) were associated with higher levels of CD3+/CD8+ lymphocytes.

One of the most important limitations of the present study is the absence of a control group. It is, therefore, a correlational study from which it is not possible to obtain causal relationships. However, though these results should be considered preliminary and need an independent confirmation and validation with a control group, the findings are strong enough to support the hypothesis of fetal transmission of the maternal inflammatory response after SARS-CoV-2 infection complicating the pregnancy.

\section{Conclusions}

We obtained data on the status of hematological and immunological parameters in maternal and umbilical cord blood in cases of SARS-CoV-2 infection during pregnancy. We could not find any traces of SARS-CoV-2 in placental samples. However, the relationship between fetal cellularity and the concentrations of humoral/cellular immunity components points to the existence of vertical transmission of an inflammatory response, observing changes in the concentration of inflammation mediators in fetal blood.

Author Contributions: Conceptualization, E.G.-M., E.G.-F., and J.S.J.-L.; methodology, E.G.-M., E.G.F., and R.C.-P.; formal analysis, E.G.-F.; investigation, C.C.-M., M.S.-A., M.B.-A., A.G.-C., L.M.-B., A.I.L.-F., B.B.-L., H.E.-N., and R.C.-P.; resources, E.G.-M. and J.S.J.-L.; data curation, L.M.-B., B.B.-L., A.G.-C., and H.E.-N.; writing-original draft preparation, E.G.-M., E.G.-F., and J.S.J.-L.; writingreview and editing, E.G.-M., E.G.-F., and J.S.J.-L.; funding acquisition, E.G.-M. E.G.-M. and E.G.-F. contributed equally, and both should be considered as first authors. All authors have read and agreed to the published version of the manuscript.

Funding: This research was funded by Ferring COVID-19 Investigational Grant Placental injury and immune reaction transmitted to the neonates in cases of SARS-CoV-2 infections during pregnancy. Study on placental and blood cord samples. The APC was funded by University of Málaga. Funding institutions did not participate in the design, recruitment, analysis, or interpretation of the results.

Institutional Review Board Statement: The study was conducted in accordance with the Declaration of Helsinki and approved by the Province Institutional Ethics Committee of Málaga (protocol code covid-neo in May 2020).

Informed Consent Statement: Informed consent was obtained from all subjects involved in the study.

Data Availability Statement: All presented data are available under reasonable request to corresponding author.

Acknowledgments: We would like to acknowledge the work of nursing and medical staff of the Obstetrics and Gynecology, and Anatomical Pathology departments of Regional University Hospital 
of Málaga. We want to thank the assistance of the Malaga-IBIMA-SSPA Provincial Biobank staff as well as the IBIMA laboratory staff.

Conflicts of Interest: The authors declare no conflict of interest. The funders had no role in the design of the study; in the collection, analyses, or interpretation of data; in the writing of the manuscript, or in the decision to publish the results.

\section{References}

1. Liu, H.; Wang, L.L.; Zhao, S.J.; Kwak-Kim, J.; Mor, G.; Liao, A.H. Why are pregnant women susceptible to COVID-19? An immunological viewpoint. J. Reprod. Immunol. 2020, 139, 103122. [CrossRef] [PubMed]

2. de Souza Silva, G.A.; da Silva, S.P.; da Costa, M.A.S.; da Silva, A.R.; de Vasconcelos Alves, R.R.; das Chagas Ângelo Mendes Tenório, F.; da Silva Melo, A.R.; de Freitas, A.C.; de Melo, C.M.L. SARS-CoV, MERS-CoV and SARS-CoV-2 infections in pregnancy and fetal development. J. Gynecol. Obstet. Human Reprod. 2020, 49, 101846. [CrossRef] [PubMed]

3. Wong, S.F.; Chow, K.M.; Leung, T.N.; Ng, W.F.; Ng, T.K.; Shek, C.C.; Ng, P.C.; Lam, P.W.Y.; Ho, L.C.; To, W.W.K.; et al. Pregnancy and perinatal outcomes of women with severe acute respiratory syndrome. Am. J. Obstet. Gynecol. 2004, 191, 292-297. [CrossRef] [PubMed]

4. Pollán, M.; Pérez-Gómez, B.; Pastor-Barriuso, R.; Oteo, J.; Hernán, M.A.; Pérez-Olmeda, M.; Sanmartín, J.L.; Fernández-García, A.; Cruz, I.; Fernández de Larrea, N.; et al. Prevalence of SARS-CoV-2 in Spain (ENE-COVID): A nationwide, population-based seroepidemiological study. Lancet 2020, 396, 535-544. [CrossRef]

5. Juan, J.; Gil, M.M.; Rong, Z.; Zhang, Y.; Yang, H.; Poon, L.C. Effect of coronavirus disease 2019 (COVID-19) on maternal, perinatal and neonatal outcome: Systematic review. Ultrasound Obstet. Gynecol. 2020, 56, 15-27. [CrossRef] [PubMed]

6. Mirbeyk, M.; Saghazadeh, A.; Rezaei, N. A systematic review of pregnant women with COVID-19 and their neonates. Arch Gynecol. Obstet. 2021, 304, 5-38. [CrossRef]

7. Galang, R.R.; Newton, S.M.; Woodworth, K.R.; Griffin, I.; Oduyebo, T.; Sancken, C.L.; Olsen, E.O.M.; Aveni, K.; Wingate, H.; Shephard, H.; et al. Risk Factors for Illness Severity Among Pregnant Women with Confirmed Severe Acute Respiratory Syndrome Coronavirus 2 Infection-Surveillance for Emerging Threats to Mothers and Babies Network, 22 State, Local, and Territorial Health Departments, 29 March 2020-5 March 2021. Clin. Infect. Dis. 2021, 73, S17-S23. [CrossRef]

8. Allotey, J.; Stallings, E.; Bonet, M.; Yap, M.; Chatterjee, S.; Kew, T.; Debenham, L.; Llavall, A.C.; Dixit, A.; Zhou, D.; et al. Clinical manifestations, risk factors, and maternal and perinatal outcomes of coronavirus disease 2019 in pregnancy: Living systematic review and meta-analysis. BMJ 2020, 370, 3320. [CrossRef]

9. Mullins, E.; Hudak, M.L.; Banerjee, J.; Getzlaff, T.; Townson, J.; Barnette, K.; Playle, R.; Perry, A.; Bourne, T.; Lees, C.C.; et al. Pregnancy and neonatal outcomes of COVID-19: Coreporting of common outcomes from PAN-COVID and AAP-SONPM registries. Ultrasound Obstet. Gynecol. 2021, 57, 573-581. [CrossRef] [PubMed]

10. Moltner, S.; de Vrijer, B.; Banner, H. Placental infarction and intrauterine growth restriction following SARS-CoV-2 infection. Arch Gynecol. Obstet. 2021, 304, 1. [CrossRef]

11. Linehan, L.; O’Donoghue, K.; Dineen, S.; White, J.; Higgins, J.R.; Fitzgerald, B. SARS-CoV-2 placentitis: An uncommon complication of maternal COVID-19. Placenta 2021, 104, 261-266. [CrossRef] [PubMed]

12. Watkins, J.C.; Torous, V.F.; Roberts, D.J. Defining severe acute respiratory syndrome coronavirus 2 (SARS-CoV-2) placentitis a report of 7 cases with confirmatory in situ hybridization, distinct histomorphologic features, and evidence of complement deposition. Arch. Pathol. Lab. Med. 2021, 145, 1341-1349. [CrossRef]

13. Schwartz, D.A.; Baldewijns, M.; Benachi, A.; Bugatti, M.; Collins, R.R.J.; de Luca, D.; Facchetti, F.; Linn, R.L.; Marcelis, L.; Morotti, D.; et al. Chronic histiocytic intervillositis with trophoblast necrosis is a risk factor associated with placental infection from coronavirus disease 2019 (COVID-19) and intrauterine maternal-fetal severe acute respiratory syndrome coronavirus 2 (SARS-CoV-2) transmission in live-born and stillborn infants. Arch. Pathol. Lab. Med. 2021, 145, 517-528. [CrossRef]

14. Torous, V.F.; Watkins, J.C.; Roberts, D.J. Response to “Diffuse trophoblast damage is the hallmark of SARS-CoV-2-associated fetal demise". Mod. Pathol. 2021, 1, 2. [CrossRef]

15. Mendoza, M.; Garcia-Ruiz, I.; Maiz, N.; Rodo, C.; Garcia-Manau, P.; Serrano, B.; Lopez-Martinez, R.M.; Balcells, J.; FernandezHidalgo, N.; Carreras, E.; et al. Pre-eclampsia-like syndrome induced by severe COVID-19: A prospective observational study. BJOG Int. J. Obstet. Gynaecol. 2020, 127, 1374-1380. [CrossRef] [PubMed]

16. Papageorghiou, A.T.; Deruelle, P.; Gunier, R.B.; Rauch, S.; García-May, P.K.; Mhatre, M.; Usman, M.A.; Abd-Elsalam, S.; Etuk, S.; Simmons, L.E.; et al. Preeclampsia and COVID-19: Results from the INTERCOVID prospective longitudinal study. Am. J. Obstet. Gynecol. 2021, 225, 289.e1. [CrossRef]

17. Chen, R.; Zhang, S.; Su, S.; Ye, H.; Shu, H. Interactions between Specific Immune Status of Pregnant Women and SARS-CoV-2 Infection. Front. Cell. Infect. Microbiol. 2021, 11, 721309. [CrossRef] [PubMed]

18. Mor, G.; Aldo, P.; Alvero, A.B. The unique immunological and microbial aspects of pregnancy. Nat. Rev. Immunol. 2017, 17, 469-482. [CrossRef]

19. Olmos-Ortiz, A.; Flores-Espinosa, P.; Mancilla-Herrera, I.; Vega-Sánchez, R.; Díaz, L.; Zaga-Clavellina, V. Innate immune cells and toll-like receptor-dependent responses at the maternal-fetal interface. Int. J. Mol. Sci. 2019, 20, 3654. [CrossRef] [PubMed] 
20. Taglauer, E.; Benarroch, Y.; Rop, K.; Barnett, E.; Sabharwal, V.; Yarrington, C.; Wachman, E.M. Consistent localization of SARSCoV-2 spike glycoprotein and ACE2 over TMPRSS2 predominance in placental villi of 15 COVID-19 positive maternal-fetal dyads. Placenta 2020, 100, 69-74. [CrossRef]

21. Fahmi, A.; Brügger, M.; Démoulins, T.; Zumkehr, B.; Oliveira Esteves, B.I.; Bracher, L.; Wotzkow, C.; Blank, F.; Thiel, V.; Baud, D.; et al. SARS-CoV-2 can infect and propagate in human placenta explants. Cell Rep. Med. 2021, 2, 100456. [CrossRef]

22. Diriba, K.; Awulachew, E.; Getu, E. The effect of coronavirus infection (SARS-CoV-2, MERS-CoV, and SARS-CoV) during pregnancy and the possibility of vertical maternal-fetal transmission: A systematic review and meta-analysis. Eur. J. Med. Res. 2020, 25, 39. [CrossRef] [PubMed]

23. Rosen, H.; Bart, Y.; Zlatkin, R.; Ben-Sira, L.; Ben Bashat, D.; Amit, S.; Cohen, C.; Regev-Yochay, G.; Yinon, Y. Fetal and Perinatal Outcome Following First and Second Trimester COVID-19 Infection: Evidence from a Prospective Cohort Study. J. Clin. Med. 2021, 10, 2152. [CrossRef] [PubMed]

24. Li, Y.; Zhao, R.; Zheng, S.; Chen, X.; Wang, J.; Sheng, X.; Zhou, J.; Cai, H.; Fang, Q.; Yu, F.; et al. Lack of Vertical Transmission of Severe Acute Respiratory Syndrome Coronavirus 2, China. Emerg. Infect. Dis. 2020, 26, 1335-1336. [CrossRef]

25. Wang, S.; Guo, L.; Chen, L.; Liu, W.; Cao, Y.; Zhang, J.; Feng, L. A Case Report of Neonatal 2019 Coronavirus Disease in China. Clin. Infect. Dis. 2020, 71, 853-857. [CrossRef]

26. Zeng, H.; Xu, C.; Fan, J.; Tang, Y.; Deng, Q.; Zhang, W.; Long, X. Antibodies in Infants Born to Mothers with COVID-19 Pneumonia. J. Am. Med. Assoc. 2020, 323, 1848-1849. [CrossRef]

27. BioNova; Diaclone Immunology Products Quick Guide Catalogue Version 6. 2020, pp. 1-24. Available online: https://www. bionova.es/documents/diaclone/diaclone_catalog_2019.pdf (accessed on 20 December 2021).

28. Biosystems Switzerland AG. BOND RNAscope Detection Reagents-Brown. Available online: https://www.biosystems.ch/ default.aspx?globalid=4103598\&lang=EN (accessed on 1 January 2022).

29. Ashary, N.; Bhide, A.; Chakraborty, P.; Colaco, S.; Mishra, A.; Chhabria, K.; Jolly, M.K.; Modi, D. Single-Cell RNA-seq Identifies Cell Subsets in Human Placenta That Highly Expresses Factors Driving Pathogenesis of SARS-CoV-2. Front. Cell Dev. Biol. 2020, 8. [CrossRef]

30. Zheng, Q.L.; Duan, T.; Jin, L.P. Single-cell RNA expression profiling of ACE2 and AXL in the human maternal-Fetal interface. Reprod. Dev. Med. 2020, 4, 7. [CrossRef]

31. Pique-Regi, R.; Romero, R.; Tarca, A.L.; Luca, F.; Xu, Y.; Alazizi, A.; Leng, Y.; Hsu, C.D.; Gomez-Lopez, N. Does the human placenta express the canonical cell entry mediators for SARS-CoV-2? Elife 2020, 9, 1-15. [CrossRef]

32. Wong, Y.P.; Khong, T.Y.; Tan, G.C. The Effects of COVID-19 on Placenta and Pregnancy: What Do We Know So Far? Diagnostics 2021, 11, 94. [CrossRef] [PubMed]

33. Waysbort, A.; Giroux, M.; Mansat, V.; Teixeira, M.; Dumas, J.C.; Puel, J. Experimental study of transplacental passage of alpha interferon by two assay techniques. Antimicrob. Agents Chemother. 1993, 37, 1232-1237. [CrossRef]

34. Yockey, L.J.; Iwasaki, A. Interferons and Proinflammatory Cytokines in Pregnancy and Fetal Development. Immunity 2018, 49, 397-412. [CrossRef] [PubMed]

35. Flannery, D.D.; Gouma, S.; Dhudasia, M.B.; Mukhopadhyay, S.; Pfeifer, M.R.; Woodford, E.C.; Triebwasser, J.E.; Gerber, J.S.; Morris, J.S.; Weirick, M.E.; et al. Assessment of Maternal and Neonatal Cord Blood SARS-CoV-2 Antibodies and Placental Transfer Ratios. JAMA Pediatr. 2021, 175, 594-600. [CrossRef] [PubMed]

36. Joseph, N.T.; Dunlop, A.L.; Patel, R.M.; Badell, M.; Dude, C. LB01 Maternal antibody response and placental antibody transfer following asymptomatic and symptomatic SARS-CoV-2 infection. Am. J. Obstet. Gynecol. 2021, 224, S722. [CrossRef] 